\title{
Galactic Cosmic Ray Variability at Two Neutron Monitors: Relation to Kp Index
}

\author{
Kingsley Chukwudi Okpala \\ University of Nigeria, Nsukka 410002, Nigeria \\ Correspondence should be addressed to Kingsley Chukwudi Okpala; kingsley.okpala@unn.edu.ng
}

Received 8 August 2014; Revised 30 November 2014; Accepted 9 February 2015

Academic Editor: José F. Valdés-Galicia

Copyright (C) 2015 Kingsley Chukwudi Okpala. This is an open access article distributed under the Creative Commons Attribution License, which permits unrestricted use, distribution, and reproduction in any medium, provided the original work is properly cited.

\begin{abstract}
The average characteristics of year-to-year variability of Galactic cosmic ray (GCR) flux measured in one mid-latitude neutron monitor stations (Newark) and high latitude station (Apatity) have been studied under different planetary disturbance (Kp) conditions. The year-to-year variability which oscillates in response to solar cycle was analyzed using Fourier technique and the amplitude of variation was obtained using data for 1980-2005. There is a noticeable trend in the difference between the amplitudes of the year-to-year variation of the two stations. The difference is highest during low Kp conditions and lowest during high Kp condition. There is generally lesser association of GCR with solar wind (SW) flow pressure and density as the Kp index increases. Similar feature is observed with the interplanetary magnetic field IMF (total). These observations have important implications for our present understanding of the effect of solar activity to variability in GCR flux.
\end{abstract}

\section{Introduction}

The variability of cosmic ray of Galactic origin near Earth is primarily driven by heliospheric factors (through the interplanetary anisotropy in the heliospheric magnetic field) and geomagnetic factors (by the geomagnetic rigidity cutoff which is latitude dependent). The natures of variability of Galactic cosmic rays (GCR) have been studied in short term scales and long term scales [1-4], and these have revealed significant information about important processes involved in the transportation of GCR from source to the neutron monitor stations. A notable factor is solar activity [58]. Neutron monitors (NM) from lower latitudes exhibited higher contribution from the 11-year phase variation which is controlled by the diffusion associated with the change in strength of the interplanetary magnetic field [9]. Kudela and Storini [10] studied the variability of cosmic rays and its association with geomagnetic activity using the disturbance storm time index (Dst) and the southward component of the heliospheric magnetic field $\left(B_{z}\right)$ component of the interplanetary magnetic field for four middle and high cutoff rigidity neutron monitors. They found high negative correlation $(-0.86)$ between GCR intensity and interplanetary magnetic field (IMF) strength for 1 month lag between the two parameters.

Understanding the nature of the variability of cosmic rays especially GCR has continued to attract research interests because of its possible effects on many geophysical processes. Many authors have studied the diurnal and semidiurnal anisotropies $[2,11]$, annual and semiannual anisotropies [6, 12], and decadal to geologic time scales of GCR flux. Cosmic rays on acceleration through the galaxy become modulated in the heliosphere by the solar wind magnetic field. The differential energy spectrum of GCR in the vicinity of the Earth can be parameterized by the field model which has only one parameter-the modulation potential for a given interstellar spectrum [13]. The geomagnetic field further modulates the GCR flux through the cut-off rigidity which can be adequately described by Stormer's equation:

$$
P_{c}=1.9 M\left(\frac{R_{o}}{R}\right)^{2} \cos ^{4} \lambda_{G}[\mathrm{GV}],
$$

where $M$ is the geomagnetic field dipole moment (in $\left.10^{25} \mathrm{Gcm}^{3}\right), R_{\mathrm{o}}$ is the Earth's mean radius, $R$ is the distance from the given location to the dipole centre, and $\lambda_{G}$ is 
the geomagnetic latitude. It is expected that changes in the dipole moment will modulate the flux of Galactic cosmic rays especially in the mid and low latitudes.

McComas et al. [14] used Ulysses and ACE observations to tie the differences in the much less variable solar wind parameters in the polar cap holes (especially density and dynamic pressure) to nearly identical variations in the ecliptic measurement. The differences seen at high latitudes are largely driven by changes in the Sun and its solar wind output and not just the differences in polar carp holes. The weaker solar wind is directly related to the lower average strength of the Sun's open magnetic field which leads to lower supply of mass and energy. The dynamic pressure carries most of the energy of the solar wind in form of bulk flow energy. Leer and Holzer [15] showed that the addition of energy above or below the sonic point is balanced by the increase (or decrease) in the wind speed and mass flux, respectively, which ultimately keeps the solar wind speed roughly the same. So it is expected that the contribution of these parameters (namely, IMF, $B_{z}$, and SW speed and density) to GCR modulation will vary in response to the level of irregularities in the heliospheric energy density.

Gleeson and Axford [16] showed that the relationship between the product of solar wind velocity and total magnetic field and the 27-day GCR intensity variation has an evident physical origin which is a hidden effect of the electric field in the cosmic ray transport [4]. Few studies have considered modulations of cosmic rays during solar activity and using certain arrangements of geomagnetic activity level (e.g., [2]), but not much work has been done to understand the association of the solar wind parameters during different levels of interplanetary disturbance. Since a wide range of processes lead to the modulation of GCR flux on the surface of the Earth, search for dominant factors under different interplanetary conditions is needed. In this paper, the GCR flux under different interplanetary condition for two stations is studied with a view to find statistical associations with solar drivers during different interplanetary conditions. The interplanetary disturbance conditions are inferred from the $\mathrm{Kp}$ index and subdivided into five groups. The Kp index is obtained from the standardized 3-hour range in the magnetic activity related to a geomagnetically quiet day curve. The $\mathrm{Kp}$ is obtained from 13 geomagnetic observatories between $44^{\circ}$ and $60^{\circ}$ north or south of the dip equator [17].

\section{Source(s) of Data}

2.1. Cosmic Ray Data. Cosmic ray data consists of pressure corrected hourly data of neutron monitor (NM) count rates from Newark $\left(39.7^{\circ} \mathrm{N}, 75.8^{\circ} \mathrm{W}\right.$, and cut-off rigidity $\left.2.4 \mathrm{GV}\right)$ and Apatity $\left(67.6^{\circ} \mathrm{N}, 33.4^{\circ} \mathrm{E}\right.$, and cut-off rigidity $\left.0.65 \mathrm{GV}\right)$. The Newark data is courtesy of Professor Beiber of the Bartol Research Institute and the Department of Physics and Astronomy, University of Delaware, USA, and the Apatity data is courtesy of Professor Eduard Vashenyuk of the Polar Geophysical Institute of the Russian Academy of Sciences. The data used was for the period 1980-2005.
2.2. Solar Wind Data. Daily mean of solar wind (SW) parameters for this study was obtained from the OMNI database through $\mathrm{ftp}$ : from OMNIweb. The solar wind parameters used are total interplanetary magnetic field IMF ( $B$ in nT), the $z$ component of the interplanetary magnetic field $B_{z}(\mathrm{nT})$, the solar wind bulk speed ( $V$ in $\mathrm{km} / \mathrm{sec}$ ) in GSE coordinate, solar wind proton density $\rho_{p}$ (number $/ \mathrm{cm}^{3}$ ), solar wind flow pressure $(P$ in $\mathrm{nPa})$, and the daily global $\mathrm{Kp}$ index. The annual means were set to zero 1990 and were linearly scaled. The global Kp index was obtained from ftp://ftp.ngdc.noaa.gov.

\section{Method of Analysis}

The data for all the days between 1980 and 2006 were divided according to their daily $\mathrm{Kp}$ index. The Kp index was divided into 5 groups, namely, Kp1 (for Kp $=0-10$ ), Kp2 (for $\mathrm{Kp}=$ $11-20), \mathrm{Kp} 3$ (for $\mathrm{Kp}=21-30), \mathrm{Kp} 4$ (for $\mathrm{Kp}=31-40)$, and $\mathrm{Kp} 5$ for $(\mathrm{Kp}>40)$. The $\mathrm{Kp}$ scale is a good indicator of global level of geomagnetic activity and has the advantage of providing quantitative measure of the level of quietness (or disturbance) associated with the solar particle radiation within the heliosphere. The greater the Kp index, the more the level of interplanetary magnetic field disturbance; hence Kp1 is equivalent to the quietest conditions in a period and Kp5 is the most disturbed conditions. Days of Forbush decreases have been removed from the analysis to avoid superposed variation that is associated with such days. Forbush decreases are sudden decreases in cosmic rays in the vicinity of the Earth usually associated with transient interplanetary events related to coronal mass ejections from the Sun and/or corotating high speed of solar wind speed. Linear regression analysis was performed on year-to-year basis to understand the effect of the different solar wind parameters on the flux of GCR at each of the NM station. The yearly averages for each group of $\mathrm{Kp}$ index were computed as means of all the days having Kp index within the range for that group. Figure 1 shows the yearly means set to zero for 1990 and scaled linearly for the two stations and for all the groups of Kp index discussed earlier.

Since GCR flux variation on an annual scale follows the solar activity oscillation, it is possible to find the harmonic amplitude of its oscillation by performing Fourier transformation to obtain the peak of the fundamental harmonic.

The time dependent harmonic function $F(t)$ with 12 equidistant points (approximating to a solar cycle) in the interval $t=0$ to $t=\pi$ can be expressed in terms of Fourier series as

$$
\begin{gathered}
F(t)=a_{o}+\sum_{n=1}^{12} a_{n} \cos (n t)+b_{n} \sin (n t), \\
F(t)=a_{o}+\sum_{n=1}^{12}\left(r_{n} \cos (n t)-\phi_{n}\right),
\end{gathered}
$$

where $a_{0}$ is the mean value of the $F(t)$ for the time interval from $t=0$ to $t=\pi, a_{n} b_{n}$ are the coefficients of the $n$th harmonics, and $r_{n}$ is the amplitude of the $n$th harmonic. For 


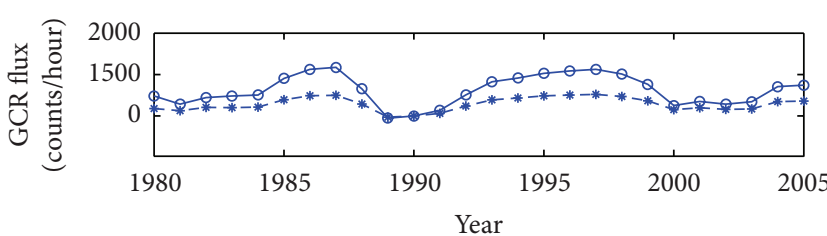

(a) for Kp1

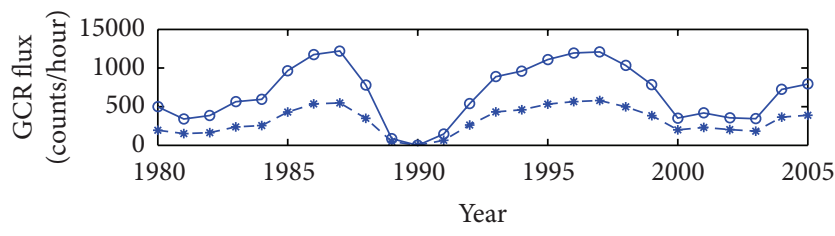

(c) for Kp3

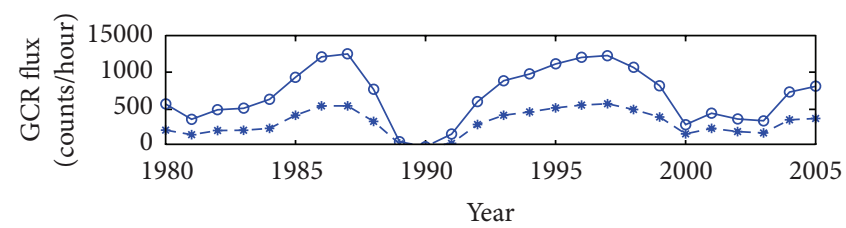

(b) for Kp2

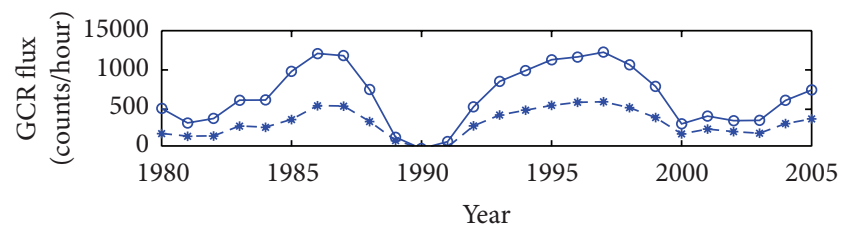

(d) for Kp4

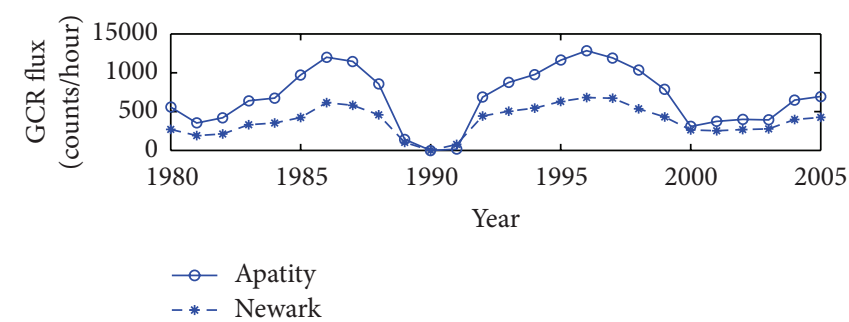

(e) for $\mathrm{Kp} 5$

FIgURE 1: Annual means of the GCR flux set to zero for 1990 and scaled linearly.

the present study the fundamental harmonic is of interest. These coefficient are expressed as

$$
\begin{gathered}
a_{o}=\frac{1}{6} \sum_{n=1}^{12} n_{i}, \\
a_{n}=\frac{1}{6} \sum_{n=1}^{12} n_{i} \cos \left(n_{i} t\right), \\
b_{n}=\frac{1}{6} \sum_{n=1}^{12} n_{i} \sin \left(n_{i} t\right) .
\end{gathered}
$$

The amplitude $r_{n}$ of the $n$th harmonic is expressed as

$$
r_{n}=\left(a_{n}^{2}+b_{n}^{2}\right)^{1 / 2} .
$$

The amplitudes for the first harmonic for Apatity $\left(r_{\text {Apatity }}\right)$ and Newark $\left(r_{\text {Newark }}\right)$ were obtained and the difference (DA) for each Kp group was computed as

$$
\mathrm{DA}=r_{\text {Apatity }}-r_{\text {Newark }}
$$

A plot of the variation of DA for the Kp groups is shown in Figure 2. The annual means of the linearly scaled count rates for $\mathrm{Kp} 1$ days for the years considered are well correlated for the two stations and are shown in Figure 3. This trend was observed for all other Kp groupings used in this study. Correlation coefficients for all the five groupings with the solar wind parameters are presented in Tables 1 and 2.

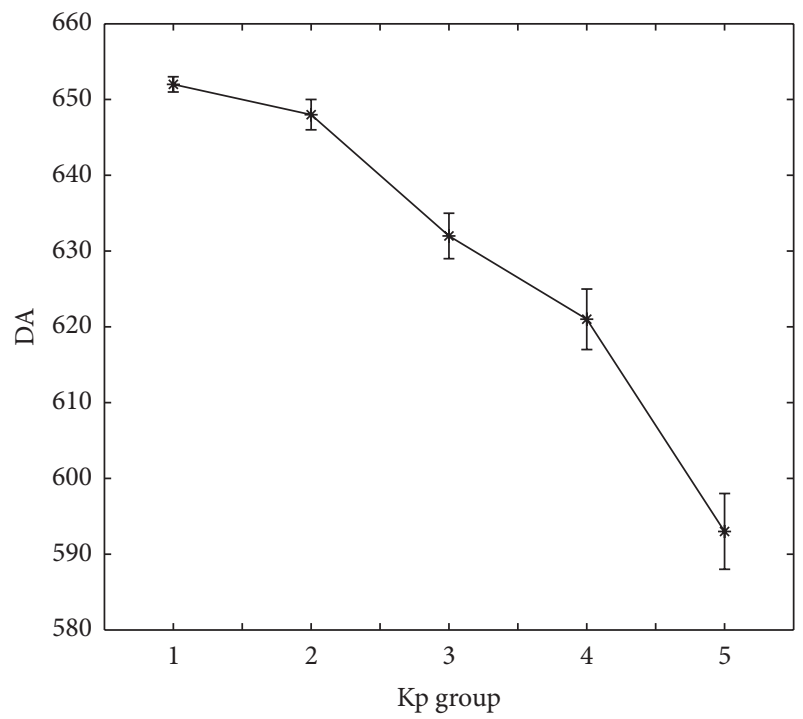

FIGURE 2: DA variation with Kp groups.

\section{Discussion of Results}

The pressure corrected hourly data of cosmic rays from two neutron monitor stations have been used to obtain the harmonic components of the annual mean of the GCR flux for over two sunspot cycles (1980-2005). Now, low Kp index days imply quiet conditions of the interplanetary medium and conversely, for high Kp index, the implication is disturbed conditions. Therefore the relationship between CR flux and 
TABLE 1: Correlation table for Apatity neutron monitor.

\begin{tabular}{cccccccc}
\hline & & IMF $(B)$ & $B_{z}$ & $\begin{array}{c}\text { SW } \\
\text { proton density }\end{array}$ & $\begin{array}{c}\text { SW } \\
\text { plasma speed }(V)\end{array}$ & $\begin{array}{c}\text { Flow } \\
\text { pressure }\end{array}$ & $\begin{array}{c}\text { Kp group } \\
\text { VB }\end{array}$ \\
\hline & -0.72 & -0.53 & 0.60 & -0.10 & 0.49 & -0.71 & $1(1164)$ \\
GCR & -0.82 & -0.68 & 0.43 & 0.03 & 0.30 & -0.81 & $2(2548)$ \\
& -0.70 & -0.59 & 0.22 & 0.26 & 0.19 & -0.65 & $3(2182)$ \\
& -0.69 & -0.06 & -0.17 & 0.28 & -0.18 & -0.69 & $4(1246)$ \\
& -0.20 & -0.13 & -0.12 & 0.13 & -0.17 & -0.27 & $5(355)$ \\
\hline
\end{tabular}

TABLE 2: Correlation table for Newark neutron monitor.

\begin{tabular}{cccccccc}
\hline & & IMF $(B)$ & $B_{z}$ & $\begin{array}{c}\text { SW } \\
\text { proton density }\end{array}$ & $\begin{array}{c}\text { SW } \\
\text { plasma speed }(V)\end{array}$ & $\begin{array}{c}\text { Flow } \\
\text { pressure }\end{array}$ & $\begin{array}{c}\text { Kp group } \\
\text { (total number of } \\
\text { days) }\end{array}$ \\
\hline \multirow{3}{*}{ GCR } & -0.77 & -0.55 & 0.61 & -0.18 & 0.48 & -0.74 & $1(1164)$ \\
& -0.85 & -0.70 & 0.47 & 0.15 & 0.37 & -0.81 & $2(2548)$ \\
& -0.71 & -0.56 & 0.28 & 0.29 & -0.26 & -0.64 & $3(2182)$ \\
& -0.56 & 0.12 & -0.08 & 0.30 & -0.15 & -0.70 & $4(1246)$ \\
& -0.47 & 0.35 & 0.03 & 0.13 & -0.50 & $5(355)$ \\
\hline
\end{tabular}

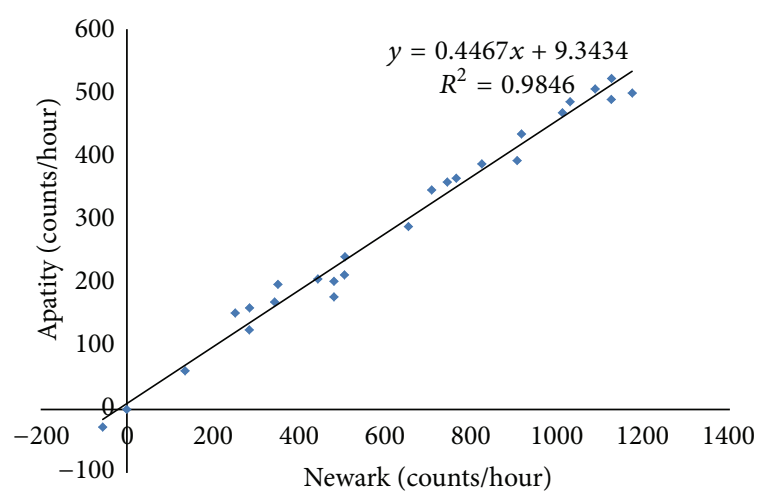

FIGURE 3: Plots of linearly scaled annual mean for Kp1 days.

interplanetary plasma parameters under varying conditions is expected to help us better appreciate the level of modulation that can be associated with key components of the solar wind under different levels of activity on an annual scale. From Figure 1, annual means of the GCR flux for Newark and Apatity showed similar annual variation following the 11-year solar cycle trend for all the levels of interplanetary disturbance with Apatity having higher amplitude because of its low rigidity. The profile of DA shown in Figure 2 as deduced from the amplitude of the Fourier analysis reveals that the difference in amplitude between the stations was lesser with increasing activity index. There appears to be a lesser variability in amplitude with increasing activity for Newark (with lower rigidity cut-off) when compared to Apatity. This trend is significant considering that the correlation between the two stations is very high (Figure 3 ). This result is particularly significant because the interplanetary disturbance is a measure of the local property of the medium through which the GCR propagates. Since GCR essentially carry signatures of various magnetohydrodynamic waves present in the interplanetary space and geospace, the variation in the two NM stations could therefore be a pointer to magnetospheric effects on the GCR flux possibly via currents such as ring currents and Birkeland currents since these currents are driven by IMF and associated bulk flows of plasma through the magnetosphere. The correlation (Tables 1 and 2) shows a similar trend in the correlation of the GCR with different SW parameters for different interplanetary condition; the difference in the amplitude determined here is likely due to changes in the rigidity cut-off of the stations. The wide variation in the DA for low Kp group of days, conversely, reflects the role of interplanetary conditions which modulate the transfer of energy into the magnetosphere leading to changes in the ever present ring current. However, the ring current (responsible for the main stage of geomagnetic storms) dominates the more disturbed conditions by reducing the rigidity cut-offs of the mid-latitude station (Newark) and consequently a decrease in DA with increasing Kp. The peaks of the annual means of the GCR showed that the highest peaks (Figure 1) are associated with Kpl while the other groups showed similar peaks though not as high as those for both Newark and Apatity stations. The amplitudes of the year-to-year variation for the two stations expressed as fraction of the mean for each group show that the least amplitude is associated with Kp1 and the highest one with Kp5. However for Apatity station, Kp2 days show a significantly high amplitude, while the same trend is not obvious for Newark.

The Newark GCR flux showed high negative correlation (at 95\% confidence) with IMF $B(-0.77)$ which tend to decrease with increasing disturbance activity. The Kp2 group showed higher correlation than the Kp1 days. This trend was also exhibited in Apatity NM (-0.72). The hidden effect of the electric field (VB component) in the transportation of GCR is evident but is mostly tied to the IMF effect rather 
than the velocity component. This can be inferred from the rather weak correlation between GCR flux and solar wind (SW) speed in the two stations considered. It is necessary to note that VB reflects both the effect of diffusion by the IMF and convection with the SW; thus its effect on the GCR modulation is expected to dominate the observations. Our study shows that this is evident, especially the mid rigidity cut-off station. The strongest association between GCR and SW speed is observed during Kp4 with no particular trend for other disturbance index grouping. It is pertinent to observe that the least correlation of GCR with $B_{z}$ (and SW proton density) coincides with the maximum correlation between GCR and SW plasma speed $(V)$. The contribution of these parameters $\left(B_{z}\right.$, SW proton density, and plasma speed) to the modulation of GCR within the heliosphere may therefore be strongly dependent on the disturbance field of the solar wind. This corroborates the findings of Leer and Holzer [15] that the addition of energy above or below the sonic point is balanced by the increase in the wind speed and mass flux, respectively, which ultimately keeps the solar wind speed roughly the same. The modulation effects by SW proton density and flow pressure on GCR flux are inversely proportional to the level of disturbance. From Tables 1 and 2, it is evident that these parameters are more associated with the GCR during quiet conditions with the SW proton density showing stronger negative association $(-0.61)$ and the flow pressure $(-0.47)$ under Kpl condition and $((-0.03)$ and $(-0.07))$ under Kp5 conditions, respectively, for Newark. The same trend is observed in Apatity NM station. The $B_{z}$ component showed fairly high correlation with GCR especially for Kp2 condition. This statistical relationship was weakest for Kp4 in the two stations.

\section{Conclusion}

(i) The difference in the amplitude of the GCR flux in Newark and Apatity NM stations depends on the level of interplanetary disturbance (Kp). The difference is highest during low $\mathrm{Kp}$ conditions and lowest during high Kp condition.

(ii) There is generally lesser association of GCR with SW flow pressure and density as the $\mathrm{Kp}$ index increases. Similar trend is observed with the total IMF.

(iii) VB and IMF (total B) correlated well with GCR flux for most of the planetary conditions considered. The correlation was least during $\mathrm{Kp} 5$ condition.

\section{Conflict of Interests}

The author declares that there is no conflict of interests regarding the publication of this paper.

\section{Acknowledgment}

The author is grateful for the many constructive suggestions from the anonymous reviewers that have greatly impacted on the final form of this paper.

\section{References}

[1] A. G. Ananth, S. P. Agrawal, and U. R. Rao, "Study of cosmic ray diurnal variation on a day-to-day basis," Pramana, vol. 3 , no. 2 , pp. 74-88, 1974.

[2] C. M. Tiwari, D. P. Tiwari, A. K. Pandey, and P. K. Shrivastava, "Average anisotropy characteristics of high energy cosmic ray particles and geomagnetic disturbance index Ap," Journal of Astrophysics and Astronomy, vol. 26, no. 4, pp. 429-434, 2005.

[3] K. C. Okpala and F. N. Okeke, "Seasonal changes in H-component of quiet day geomagnetic field modulation of galactic cosmic rays," Nigerian Journal of Space Research, vol. 7, pp. 8694, 2010.

[4] R. Modzelewska and M. V. Alania, "The 27-day cosmic ray intensity variations during solar minimum 23/24," Solar Physics, vol. 286, no. 2, pp. 593-607, 2013.

[5] H. Mavromichalaki and E. Paouris, "Long-term cosmic ray variability and the CME-index," Advances in Astronomy, vol. 2012, Article ID 607172, 8 pages, 2012.

[6] K. C. Okpala and F. N. Okeke, "Investigation of diurnal and seasonal galactic cosmic ray variations on quiet days in two mid latitude stations," Astroparticle Physics, vol. 34, no. 12, pp. 878$885,2011$.

[7] A. V. Belov, E. A. Eroshenko, V. A. Oleneva, V. G. Yanke, and H. Mavromichalki, "Long-term behaviour of the cosmic ray anisotropy derived from the worldwide neutron monitor network data," in Proceedings of the 20th ECRS, 2006.

[8] I. Usoskin, H. Kanamen, K. Mursula, P. Tanskanen, and G. A. Kovaltsov, "Correlative study of solar activity and cosmic ray intensity," Journal of Geophysical Research, vol. 103, pp. 95679574, 1998.

[9] S. Y. Oh, Y. Yi, and J. W. Bieber, "Modulation cycles of galactic cosmic ray diurnal anisotropy variation," Solar Physics, vol. 262, no. 1, pp. 199-212, 2010.

[10] K. Kudela and M. Storini, "Cosmic ray variability and geomagnetic activity: a statistical study," Journal of Atmospheric and Solar-Terrestrial Physics, vol. 67, no. 10, pp. 907-912, 2005.

[11] K. Munakata, T. Kitawada, S. Yasue et al., "Enhanced sidereal diurnal variation of galactic cosmic rays observed by the twohemisphere network of surface level muon telescopes," Journal of Geophysical Research A: Space Physics, vol. 104, no. 2, pp. 25112519, 1999.

[12] S. Oh, J. W. Bieber, P. Evenson, J. Clem, Y. Yi, and Y. Kim, "Record neutron monitor counting rates from galactic cosmic rays," Journal of Geophysical Research: Space Physics, vol. 118, no. 9, pp. 5431-5436, 2013.

[13] I. G. Usoskin, K. Alanko-Huotari, G. A. Kovaltsov, and K. Mursula, "Heliospheric modulation of cosmic rays: monthly reconstruction for 1951-2004," Journal of Geophysical Research, vol. 110, no. A12, p. A12108, 2005.

[14] D. J. McComas, R. W. Ebert, H. A. Elliott et al., "Weaker solar wind from the polar coronal holes and the whole sun," Geophysical Research Letters, vol. 35, no. 18, Article ID L18013, 2008.

[15] E. Leer and T. E. Holzer, "Energy addition in the solar wind," Journal of Geophysical Research, vol. 85, no. A9, pp. 4681-4688, 1980.

[16] L. J. Gleeson and W. I. Axford, "Cosmic rays in the interplanetary medium," The Astrophysical Journal, vol. 149, p. L115, 1967.

[17] NGDC, Monthly Summary of Geomagnetic Activity, Issue 2, Geomagnetic Indices Bulletin, 1985. 

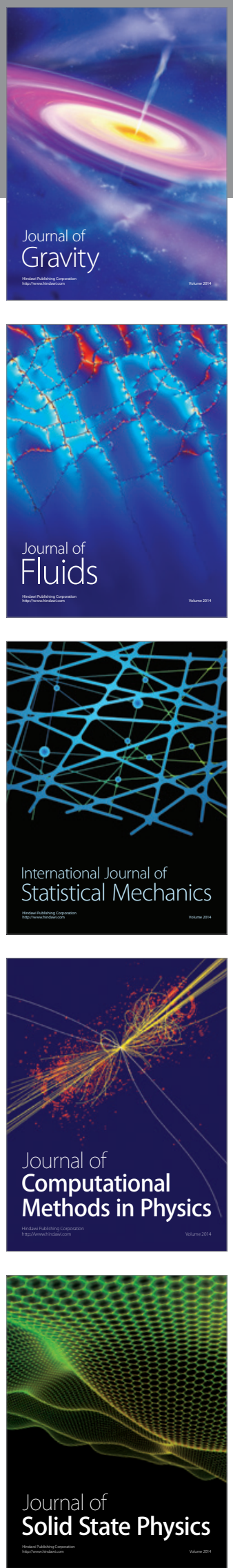

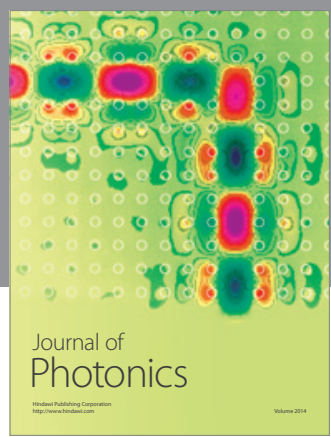

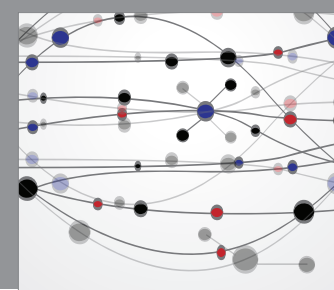

The Scientific World Journal

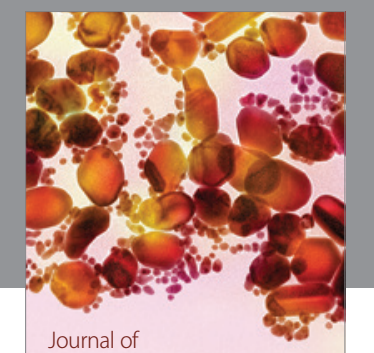

Soft Matter
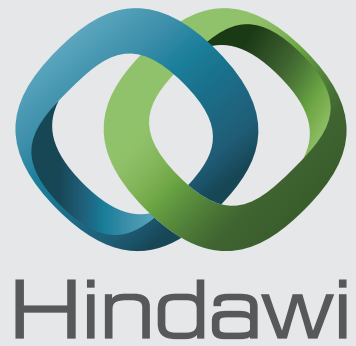

Submit your manuscripts at

http://www.hindawi.com
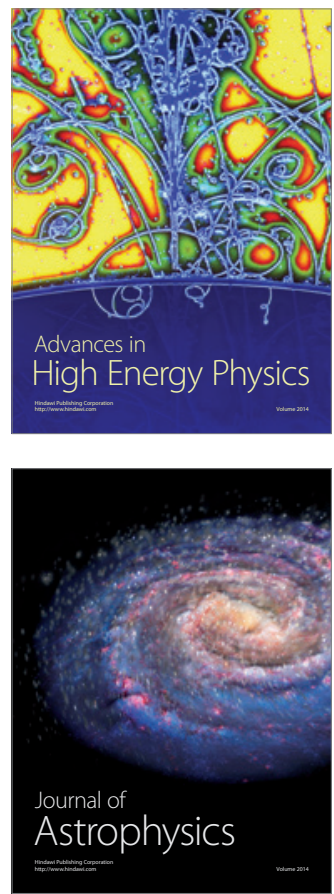
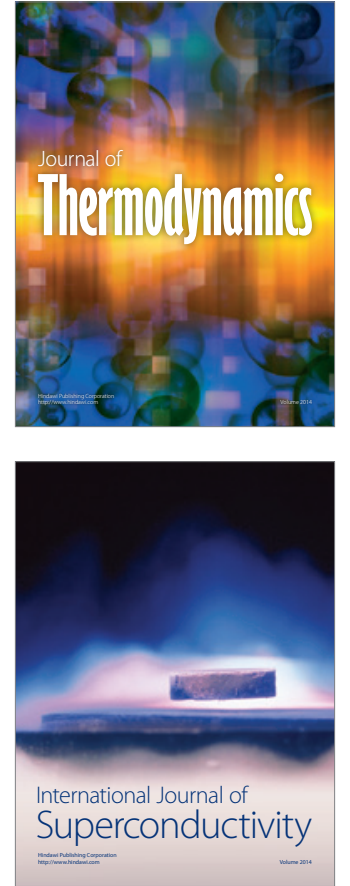
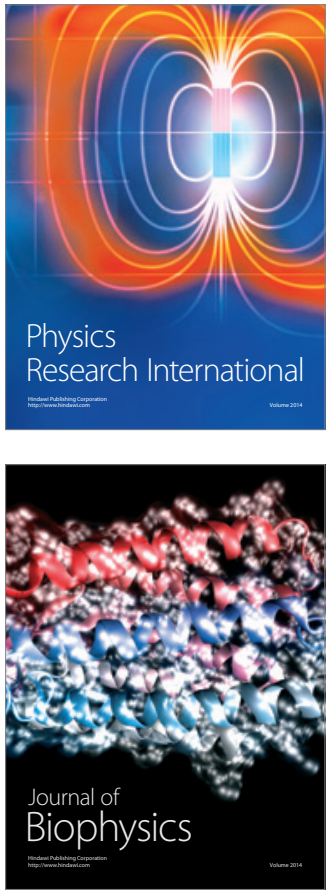
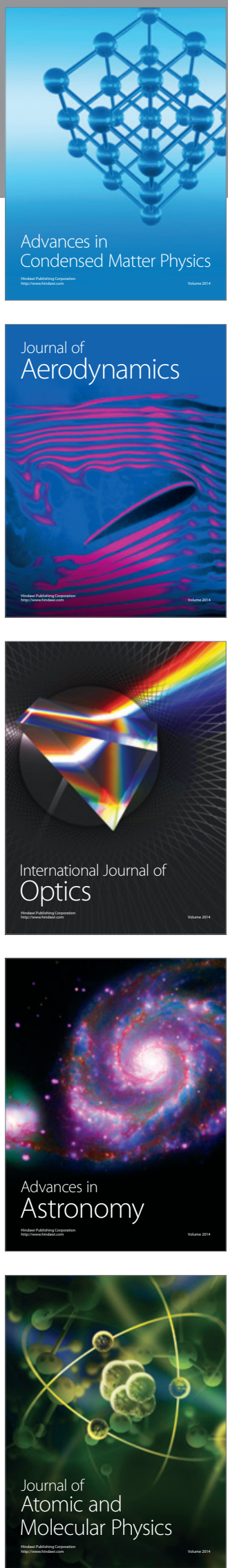